\title{
La formación de niños como investigadores en la feria de las ciencias y creatividad de Coahuila
}

\section{The training of children as researchers at the Coahuila science and creativity fair}

\author{
MUÑOZ-LÓPEZ, Temístoclesł**, RAMOS-JAUBERT Rocío I., ESPERICUETA-MEDINA, Marta \\ Nieves y MARTÍNEZ-SOSA Vanessa
}

Universidad Autónoma de Coahuila, Faculta de Ciencia, Educación y Humanidades, México.

ID $1^{\mathrm{er}}$ Autor: Temístocles, Muñoz-López / ORC ID: 0000-0003-4940-5730, Web of Science Researcher ID: X-7834-2018, CVU CONACYT ID: 202437

ID $1^{\text {er }}$ Coautor: Rocío I., Ramos-Jaubert / ORC ID: 0000-0003-3289-5390, Web of Science Researcher ID: T-1652-2018, CVU CONACYT ID: 201861

ID $2^{\text {do }}$ Coautor: Marta Nieves, Espericueta-Medina / ORC ID: 0000-0002-4924-7632, Web of Science Researcher ID: T1500-2018, CVU CONACYT ID: 372705

ID $3^{\text {er }}$ Coautor: Vanessa, Martínez-Sosa / ORC ID: 0000-0001-9442-8363, CVU CONACYT ID: 333696

DOI: $10.35429 / J B E .2020 .12 .4 .8 .18$

Recibido 15 de Julio, 2020; Aceptado 30 de Diciembre, 2020

Resumen

El presente trabajo es un estudio de niños de cuarto, quinto y sexto año de primaria que realizaron proyectos de investigación para la feria de ciencias y creatividad 2018 que organiza el Consejo estatal de Ciencia y Tecnología de Coahuila en coordinación con el CONACyT. La investigación se realizó con el propósito de tener un perfil del proceso de investigación que realizan los niños cuando se le solicita elaborar un proyecto para presentarlo en la feria. Los resultados de los proyectos fueron evaluados por 17 evaluadores e investigadores seleccionados por el Consejo Estatal de Ciencia y Tecnología con formatos preestablecidos en escalas tipo Likert y clasificados en los tipos científicos, tecnológicos y ciudadanos. Las escalas originales fueron transformadas con un método estandarizado de la versión española del Cuestionario de Salud SF-36 para trabajar los datos en escala de cero al 100, de tal forma que se pudieran realizar análisis multi variados como análisis discriminante y análisis factorial. Los resultados muestran un perfil de los niños investigadores donde existen ocho variables que constituyen le estructura básica como investigadores y un perfil complementario que refiere a la información y comunicación del resultado de sus proyectos. Estas variables de su perfil básico son: Identifica problemas, tiene Claridad sobre el Proyecto, Recaba datos apropiadamente, Realiza y registra observaciones de campo, Planea y lleva a cabo una investigación en el medio local, con un propósito definido, Relaciona sus aprendizajes con la vida cotidiana, Utiliza fuentes de información confiable, y Desarrolla nuevos conocimientos.

Investigación en niños, Habilidades de investigación, Formación de investigadores

\begin{abstract}
This work is a study of children in the fourth, fifth and sixth years of primary school who carried out research projects for the 2018 science and creativity fair organized by the State Council of Science and Technology of Coahuila in coordination with CONACyT. The research was carried out with the purpose of having a profile of the research process that children carry out when they are asked to develop a project to present it at the fair. The results of the projects were evaluated by 17 evaluators and researchers selected by the State Council for Science and Technology with pre-established formats on Likert-type scales and classified into scientific, technological and citizen types. The original scales were transformed with a standardized method of the Spanish version of the SF-36 Health Questionnaire to work the data on a scale from zero to 100 , in such a way that multivariate analyzes such as discriminant analysis and factor analysis could be performed. The results show a profile of child researchers where there are eight variables that constitute the basic structure as researchers and a complementary profile that refers to the information and communication of the results of their projects. These variables of his basic profile are: Identify problems, have Clarity about the Project, collect data appropriately, Make and record field observations, Plan and carry out an investigation in the local environment, with a defined purpose, relate their learning to the everyday life, uses reliable sources of information, and develops new knowledge.
\end{abstract}

Research in children, Research skills, Training of researchers

Citación: MUÑOZ-LÓPEZ, Temístocles, RAMOS-JAUBERT Rocío I., ESPERICUETA-MEDINA, Marta Nieves y MARTÍNEZ-SOSA Vanessa. La formación de niños como investigadores en la feria de las ciencias y creatividad de Coahuila. Revista de Educación Básica. 2020. 4-12:8-18.

*Correspondencia al Autor (Correo Electrónico: temistoclesmunoz@adec.edu.mx)

$\dagger$ Investigador contribuyendo como primer autor. 


\section{Introducción}

La Feria de Ciencias y Creatividad es un concurso de proyectos de estudiantes y maestros de primaria a inscribir proyectos Científicos, Tecnológicos y/o Ciudadanos relacionados con los ámbitos de la asignatura de Ciencias Naturales que marcan los programas de la Secretaría de Educación. Su convocatoria establece que surge como parte del programa Apropiación Social de la Ciencia, Tecnología e Innovación, el Consejo Estatal de Ciencia y Tecnología de Coahuila de Zaragoza, con el apoyo del Consejo Nacional de Ciencia y Tecnología, con fundamento en el Programa Especial de Ciencia, Tecnología e Innovación (PECITI) 2014 - 2018, y con el objeto de impulsar el pensamiento científico, la creatividad y la innovación entre los estudiantes de los tres últimos grados de primaria, así como fomentar las vocaciones científicas en Coahuila.

El Programa recibe tres modalidades de trabajo por proyectos: a) Proyectos Científicos, dirigidos a la realización de actividades en las que se describan, expliquen y/o predigan fenómenos o procesos naturales que ocurren en nuestro entorno, promoviendo la curiosidad intelectual, la investigación, perseverancia, escepticismo informado y creatividad.; b) Proyectos Tecnológicos que promuevan el uso sistemático del conocimiento y la investigación encaminado al diseño y construcción de objetos técnicos, producción de materiales y herramientas, dispositivos, sistemas o métodos; incluyendo el diseño, desarrollo, mejora de prototipos, procesos, productos, servicios o modelos organizativos; c) Proyectos Ciudadanos, como propuestas de proyectos que fomenten y fortalezcan las relaciones ente la ciencia y la sociedad, mediante una dinámica de investigación-acción, con el objetivo de brindar la oportunidad de identificar y analizar problemas sociales, reconocer las dificultades y proponer alternativas de solución viables.

\section{Objetivos}

Esta investigación pretende obtener el perfil de los niños que actúan como investigadores en la Feria de Ciencias y Creatividad para denotar sus puntos fuertes y déficits metodológicos, de tal manera que esto permita mejorar sus capacidades y habilidades para investigar e innovar, al tiempo que se estructure el proceso de investigación que normalmente utilizan.
De esta forma se espera mejorar también la cantidad y calidad de trabajos que sean presentados en la feria de la ciencia y la creatividad y la formación de los maestros asesores de los niños de primaria.

\section{Perspectiva teórica}

Existen una gran cantidad de perspectivas que abordan la formación de los niños como investigadores, como un elemento educativo que les permite sistematizar mejor sus experiencias de vida y de aprendizaje. El libro de "Los niños y la ciencia: La aventura de la mano en la masa" cuenta la historia de una experiencia pedagógica que convirtió a los chicos en investigadores y a las aulas en laboratorios. Ahí comentan los autores que debemos adoptar el proceso de la construcción de los conocimientos científicos como el principal componente en la educación científica de los niños en nuestros países (Charpak, Léna, \& Quéré, 2006) lo que conlleva al impulso de una cultura de innovación mediante los proyectos de los estudiantes que realizan tareas interdisciplinarias, y que además aplicarán su conocimiento y creatividad en el mundo real.

Luisa Massarani (Massarani, L., 2007) menciona que la práctica de la ciencia, tal como la realizamos los científicos en nuestros laboratorios, es la mejor manera de aprender, no solo conocimientos, si no también valores, actitudes y comportamientos que forman mejores ciudadanos y mejores personas. Esa idea implica que debemos adoptar el proceso de la construcción de los conocimientos científicos como el principal componente en la educación científica de los niños en nuestros países de acuerdo con Charpak, et Al. (Op. Cit).

$\begin{array}{ccc}\begin{array}{c}\text { Insiste en que los niños } \\ \text { extremadamente }\end{array} & \text { son } \\ \text { curiosos } & \text { acerca } & \text { del }\end{array}$ funcionamiento de las cosas y del mundo que les rodea y en que vale la pena preguntarse si esa característica ha sido suficientemente explorada. En general, los contenidos científicos para niños son de calidad y presentación inadecuados, no permiten el establecimiento de relaciones significativas con el entorno y no favorecen la adquisición de una visión más clara de la actividad científica, con sus ventajas y limitaciones. 
Frutos Marazuela, de la Facultad de Educación y Trabajo Social. Universidad de Valladolid (Frutos Marazuela, 2013), comenta varias cualidades de los niños en la investigación, donde destacan las siguientes:

Los niños durante el proceso de investigación, deben de comunicarse entre ellos, por lo que se van estableciendo normas sociales de comunicación y comportamiento para con los demás niños y para con los adultos, ya que también interrelacionan con el profesor.

Se obtiene la Competencia en el conocimiento y la interacción con el mundo físico. Pretende que los estudiantes amplíen su conocimiento de las personas, los objetos y otros elementos que conforman el mundo que les rodea, desarrollando las habilidades y destrezas necesarias para interpretar la realidad.

Tanto la metodología como el tema elegido se relacionan íntimamente con esta competencia, ya que el método científico no es otra cosa que, mediante pasos, descubrir el mundo.

Dentro de las actividades infantiles, una de las favoritas para los niños, suelen ser los experimentos. A través de los "experimentos para niños", los pequeños logran por sí solos, descubrir conceptos y verdades propias de su alrededor, de su entorno. Y no sólo eso, sino que se conciencian de la importancia que tiene el cuidado y la protección del planeta para la vida.

Los niños son curiosos por naturaleza, son "los mejores investigadores", y desde edades muy tempranas ponen en funcionamiento estrategias experimentales.

Poner a los niños en actitud de indagar sobre lo que suscita su curiosidad puede ser el conocimiento de despertar una actitud científica y un espíritu crítico ante la vida.
El aprendizaje científico es un proceso que nace de la curiosidad por conocer y comprender los fenómenos que nos rodean, los niños tienen el don innato de la curiosidad.

Así pues, enseñar las bases de la ciencia implica, además del conocimiento específico de la materia, la necesidad de una actitud científica escudriñadora pero, principalmente, exige de los educadores infantiles una actitud positiva hacia la vida por dos razones fundamentales: porque tienen en sus manos lo más preciado y precioso de nuestra sociedad, nuestros niños y porque el mundo que nos rodea está hecho de seres vivos, de sucesos naturales que pueden explicarse y de situaciones que conjugan la naturaleza física y la humana. Si los niños son la inteligencia más fresca, natural y creativa de nuestra sociedad, tratémosles con la deferencia que se merecen, con la sensibilidad y la ternura que su sola presencia nos motiva.

La investigadora continúa afirmando que la escuela debe iniciar a niños en el hábito de la indagación, en la formulación de preguntas surgidas del interés. Nuestros estudiantes están llamados ser "aprendices permanentes", a seguir sus impulsos, a investigar y a poner su energía al servicio de la acción. Partiendo de que niños interpretan los fenómenos en forma intuitiva $\mathrm{y}$ concreta, y de que sus interpretaciones se basan en el sentido común, al enseñarles el método científico, les permitimos resolver cualquier situación problemática de la vida cotidiana con ojos curiosos, mentes reflexivas y orden en el proceder.

La autora De Álvaro Marciel (2017) concuerda con otros autores asumiendo que el objetivo principal de un rincón dedicado a la experimentación es que el alumnado pueda manipular y explorar libremente todo aquello que le rodea. Los niños y niñas nacen con la necesidad de explorar el entorno en el que viven, por lo que les debemos facilitar material para que investiguen y creen sus propias experiencias. Como expresa Freinet, hay que salvaguardar en los niños, después de haber activado su curiosidad natural, el deseo innato de buscar, experimentar y crear (Sugrañes et al., 2010) y propone su proyecto está basado en los siguientes principios metodológicos: 
Interdisciplinariedad, b) Socialización, c) Aprendizaje significativo, d) Observación y experimentación, e) Curiosidad y motivación, y f) Evaluación.

Por otra parte, en un análisis general de la enseñanza y el aprendizaje de las ciencias y la investigación científica en las escuelas primarias de México, Cuevas, A., Hernández, R., Leal, B. E. y Mendoza, C. P. (2016) consideraron a 1,559 estudiantes, 74 maestros y 35 directores de 35 escuelas primarias públicas y privadas en 14 ciudades del país, los resultados apuntan a una perspectiva a la vez alentadora y exigente: alentadora porque los estudiantes muestran una actitud positiva y una visión favorable de la ciencia, y los docentes y directores muestran interés en la investigación docente; pero también exigente porque se identificaron algunas áreas que necesitaban fortalecerse, tales como la importancia de la transversalidad en la enseñanza de la investigación, la pertinencia de las actividades para la enseñanza-aprendizaje, el aprovechamiento e incorporación de actividades de educación científica informal y la gestión educativa. Su parte, Brenda Elizabeth Leal (et al. 2014) en su trabajo sobre "Actitudes asociadas a la ciencia en la educación básica en México" argumentan que las características generales de las ideas de los estudiantes se pueden enunciar como las siguientes:

- Las ideas de los estudiantes como esquemas activos no pueden cambiarse fácilmente mediante la instrucción. Podrían calificarse mejor como una serie de posibles «modos-de-ver» de que disponen los estudiantes y que pueden ser ensayadas en situaciones novedosas, son herramientas para aprender y forman una base para adquirir nuevas comprensiones mediante una forma de razonamiento analógico.

Las ideas de los estudiantes son coherentes dentro de su modo de pensar, y las concepciones que usan pueden ser coherentes vistas desde la perspectiva del niño.

El razonamiento está ligado a un contexto especifico, advertimos sobre las ideas de los niños, es que un individuo puede responder a la misma situación usando diferentes ideas en diferentes ocasiones.
Diferenciación de ideas. El carácter indiferenciado de algunas nociones permite que los niños pasen de un significado o aspecto a otro sin ser necesariamente conscientes de ello.

Del pensamiento perceptivo al conceptual en los niños más jóvenes advertimos que sus nociones tienden a estar dominadas por las percepciones; cuando una sustancia se quema la materia desaparece, cuando bebes con una pajita el líquido sube a la boca a causa de la «succión».

Atención a las propiedades más que a las interacciones en los niños pequeños se caracteriza también por una tendencia a dar interpretaciones en términos de propiedades de los objetos antes que en términos de interacciones entre sistemas.

Razonamiento causal induce a el razonamiento de los niños se centra sobre estados cambiantes más que en estados de equilibrio, esto es, es la tendencia a pensar en direcciones preferentes más que percibiendo la simetría en las interacciones entre sistemas. el razonamiento de los niños tiende a seguir una secuencia causal lineal; así, los procesos que un científico considera de una manera reversible no son necesariamente vistos de esta manera por los niños.

En otra perspectiva, su publicación de Batanero sobre "La comprensión de la probabilidad en los niños: ¿qué podemos aprender de la investigación?" se describen los resultados con el fin de orientar adecuadamente a los profesores para enseñar la probabilidad a niños en la Educación Primaria. Indica que la enseñanza ha de cuidar también las creencias infundadas sobre los experimentos aleatorios, por ejemplo, la creencia en la suerte o en números favoritos o la preferencia por un color. En el escrito refiere a Godino, Batanero y Cañizares (1987) donde se pueden consultar propuestas didácticas sobre probabilidad, basadas en dichos trabajos y para diferentes edades en la educación primaria. 
Considera como otro recurso importante la simulación que permite, mediante el apoyo de la tecnología, que los niños experimenten situaciones aleatorias y, de este modo, ganen experiencia, mejorando sus intuiciones sobre estas experiencias (Batanero, C., 2013).

También Postigo y Greca (2014) contribuyen al introducir la metodología de la indagación en la escuela primaria, que como cualquier innovación metodológica, supone un reto. La propuesta que presentan, así como los resultados de su implementación parecen mostrar que no solamente es viable - con el tiempo y recursos disponibles y los temas incluidos en el currículo oficial - sino que los niños parecen aprender mejor, además de sentirse más motivados hacia las ciencias.

No obstante, en estos esfuerzos de alfabetizar a todos los estudiantes en la ciencia, Morris H. Shamos (1995) argumenta que una significativa alfabetización científica no puede lograrse, en primer lugar, y el intento es un mal uso de los recursos humanos a gran escala. Es escéptico sobre los pronósticos de "déficits críticos en la mano de obra científica" y sobre los motivos detrás de los programas de choque para lograr que más jóvenes ingresen a la línea científica.

\section{Metodología}

Metodológicamente se abordaron dos problemas, la solución de la interrogante de investigación y la transformación de los datos capturados en los formatos preestablecidos para explotar la información, esto es, transformar la escala tipo Likert que es su baremo (normas establecidas convencionalmente para evaluar) a una escala centesimal para poder aplicar métodos multivariados.
En la base de datos están los resultados de 105 niños con valores asignados por 17 evaluadores que en la práctica son investigadores seleccionados por el Consejo Estatal de Ciencia y Tecnología, en reconocimiento a su demostrada capacidad técnica, de lo que tenemos en los resultados 33 Variables, qué fueron transformadas para lograr la recodificación de ellas con la que fueron procesadas para obtener una escala centesimal y tratamientos más precisos de análisis multivariado, de acuerdo con el Manual de puntuación de la versión española del Cuestionario de Salud SF-36 de la Unitat de Recerca en Serveis Sanitaris Institut Municipal d'Investigació Mèdica (Investigació Mèdica, I. M. (1996).

\section{Variables}

Se analizaron los datos Signalécticos como la Información general de participantes, el Tipo de Proyecto presentado por los niños, el Nombre del proyecto, el Asesor, Nombre de la escuela, Tipo de institución (1-Pública, 2-Privada), Municipio, el Grado de estudio de los participantes. Las 24 Variables (4 categorías y 20 variables simples) del proceso de investigación que integran los formatos oficiales de la Feria de Ciencias y Creatividad, requirieron ser estandarizadas en el caso de que tuviesen alguna equivalencia metodológica, por ejemplo en las habilidades para investigación científica de cada uno de los tres tipos de proyecto, o en las variables de la comunicación de los resultados del proyecto como se observa en la Tabla 4. Tabla 4. Variables evaluadas en cada uno de los tres tipos de proyectos, ciudadano, tecnológico o científico, donde se muestran las equivalencias. Se observan en la columna 2 , con una letra mayúscula antes del nombre, su correspondencia a las diferentes categorías. 


\section{Resultados}

\section{Estadística descriptiva}

Las 20 variables simples incluidas en los formatos tienen una calificación promedio máxima de 86 y una mínima de 72 . La Media de las medias es de 77.99 y la Mediana 77.95, con una Desviación estándar de 3.89 entre las medias, lo que da un Coeficiente de Variabilidad de 4.99 con un valor $\mathrm{Z}$ de 20.04, por lo que la media más una Desviación estándar es 81.88 y menos una Desviación estándar es 74.10. (ver Tabla 1).

Tabla 1. Estadísticas generales de todas las variables de acuerdo con el promedio obtenido por todos sus casos, lo que denota su grado de importancia en la perspectiva científica del niño

\begin{tabular}{|c|c|}
\hline Variables organizadas por categoría & Promedios \\
\hline Identifica problemas & 81.14 \\
\hline $\begin{array}{l}\text { Define en qué consististe el proyecto y a quien está } \\
\text { dirigido o a que sector } \\
\text { impactará positivamente }\end{array}$ & 74.76 \\
\hline $\begin{array}{l}\text { Explica cómo el objetivo será alcanzado por medio } \\
\text { del desarrollo del proyecto }\end{array}$ & 74.33 \\
\hline Recaba datos apropiadamente & 72.69 \\
\hline Realiza y registra observaciones de campo & 71.61 \\
\hline Cat. Habilidades Inv. Cient. & 74.9 \\
\hline $\begin{array}{l}\text { Planea y lleva a cabo una investigación en el medio } \\
\text { local, con un propósito } \\
\text { definido }\end{array}$ & 77.08 \\
\hline Relaciona sus aprendizajes con la vida cotidiana & 80.08 \\
\hline $\begin{array}{l}\text { Argumenta utilizando términos científicos de } \\
\text { manera adecuada }\end{array}$ & 74.29 \\
\hline Utiliza fuentes de información confiable & 74.26 \\
\hline Desarrolla nuevos conocimientos & 74.24 \\
\hline Cat. Comprensión Cient. & 76 \\
\hline Identifica lo que se necesita saber & 79.76 \\
\hline Aprende a buscar & 80.73 \\
\hline $\begin{array}{l}\text { Identifica, evalúa, selecciona, organiza y } \\
\text { sistematiza la información recolectada }\end{array}$ & 77.34 \\
\hline Se apropia de la información de manera crítica & 76.43 \\
\hline Utiliza y comparte información con sentido ético & 81.20 \\
\hline Cat. Manejo de Información & 79.6 \\
\hline $\begin{array}{l}\text { El cartel o stand presenta una organización lógica } \\
\text { de contenidos }\end{array}$ & 86.29 \\
\hline $\begin{array}{l}\text { En la presentación oral, da respuestas claras, } \\
\text { concisas y reflexivas }\end{array}$ & 83.45 \\
\hline Explica cómo impactará el producto & 80.51 \\
\hline Comunica resultados apropiadamente & 80.99 \\
\hline $\begin{array}{l}\text { Elabora conclusiones con base en la evidencia } \\
\text { disponible }\end{array}$ & 78.55 \\
\hline Cat. Comunicación & 81.96 \\
\hline Total & 20 \\
\hline Max & 86 \\
\hline Min & 72 \\
\hline Rango & 15 \\
\hline Media & 77.99 \\
\hline Mediana & 77.95 \\
\hline Desv Std & 3.89 \\
\hline $\mathrm{CV}$ & 4.99 \\
\hline$Z$ & 20.04 \\
\hline $\mathrm{N}+1$ & 81.88 \\
\hline $\mathrm{N}-1$ & 74.10 \\
\hline
\end{tabular}

Esto implica que las calificaciones más altas obtenidas por los niños corresponden a la comunicación, específicamente las variables de la presentación oral, da respuestas claras, concisas y reflexivas, y en la variable El cartel o stand presenta una organización lógica de contenidos. Bajo la normalidad a una desviación estándar encontramos con las más bajas calificaciones las variables que miden que el niño Recaba datos apropiadamente y que Realiza y registra observaciones de campo. Esto resulta interesante ya que ambas son previas a las pruebas experimentales. Todas las variables mostraron una gran consistencia dado que sus valores de $\mathrm{Z}$ fueron mayores a 1.96 como se observa en la Tabla 2.

Tabla 2. Estadísticas generales de todas las variables de acuerdo con el promedio obtenido por todos sus casos, lo que denota su grado de importancia en la perspectiva científica del niño.

\begin{tabular}{|l|r|}
\hline Variables organizadas por categoría & Promedios \\
\hline \begin{tabular}{l} 
Identifica problemas \\
\hline $\begin{array}{l}\text { Define en qué consististe el proyecto y a } \\
\text { quien está dirigido o a que sector impactará } \\
\text { positivamente }\end{array}$
\end{tabular} \\
\hline $\begin{array}{l}\text { Explica cómo el objetivo será alcanzado por } \\
\text { medio del desarrollo del proyecto }\end{array}$ & 74.76 \\
\hline Recaba datos apropiadamente & 74.33 \\
\hline Realiza y registra observaciones de campo & 72.69 \\
\hline Cat. Habilidades Inv. Cient. & 71.61 \\
\hline $\begin{array}{l}\text { Planea y lleva a cabo una investigación en } \\
\text { el medio local, con un propósito definido }\end{array}$ & 74.9 \\
\hline $\begin{array}{l}\text { Relaciona sus aprendizajes con la vida } \\
\text { cotidiana }\end{array}$ & 77.08 \\
\hline $\begin{array}{l}\text { Argumenta utilizando términos científicos de } \\
\text { manera adecuada }\end{array}$ & 80.08 \\
\hline Utiliza fuentes de información confiable \\
\hline Desarrolla nuevos conocimientos & 74.29 \\
\hline Cat. Comprensión Cient. & 74.26 \\
\hline Identifica lo que se necesita saber & 74.24 \\
\hline Aprende a buscar & 76 \\
\hline $\begin{array}{l}\text { Identifica, evalúa, selecciona, organiza y } \\
\text { sistematiza la información recolectada }\end{array}$ & 79.76 \\
\hline $\begin{array}{l}\text { Se apropia de la información de manera } \\
\text { crítica }\end{array}$ & 80.73 \\
\hline $\begin{array}{l}\text { Utiliza y comparte información con sentido } \\
\text { ético }\end{array}$ & 77.34 \\
\hline Cat. Manejo de Información & 81.20 \\
\hline $\begin{array}{l}\text { El cartel o stand presenta una organización } \\
\text { lógica de contenidos }\end{array}$ & 86.43 \\
\hline $\begin{array}{l}\text { En la presentación oral, da respuestas } \\
\text { claras, concisas y reflexivas }\end{array}$ \\
\hline Explica cómo impactará el producto \\
\hline Comunica resultados apropiadamente \\
\hline $\begin{array}{l}\text { Elabora conclusiones con base en la } \\
\text { evidencia disponible }\end{array}$ \\
\hline Cat. Comunicación \\
\hline
\end{tabular}

Donde: Total: 20: Max: 86: Min: 72: Rango: 15: Media: 77.99: Mediana: 77.95: Desv Std: 3.89: CV: 4.99: Z: 20.04: N+1: 81.88: N-1: 74.10 


\section{Análisis de Correlación}

El análisis correlacional separa de forma natural los cuatro grupos de categorías, las Habilidades de Investigación Científica, la Comprensión de fenómenos y procesos naturales desde la perspectiva científica, el Manejo de Información y la Comunicación de resultados del Proyecto.

En adelante, en la lectura de los resultados se ponen con mayúscula inicial los nombres de las variables en estudio por considerarlas nombres propios.

Las Habilidades Investigación Científica es una categoría fundamental para construir sobre ella al futuro investigador, por lo cual es interesante observar que en ella existen los elementos en la formación de los niños, sin embargo no están articulados entre sí como fuera deseable para que pudiéramos considerarlos una plataforma de desarrollo de posteriores trabajos de investigación de los niños en secundaria. Se denota que si existe una relación entre su capacidad de Identificar problemas y tener Claridad en su proyecto de investigación, en tanto que el Recabar datos apropiadamente y Registrar observaciones de campo también están articulados de una manera significativa, quedando separada la Constatación empírica que normalmente se hace en la investigación tras el planteamiento de las hipótesis (

Figura 1).

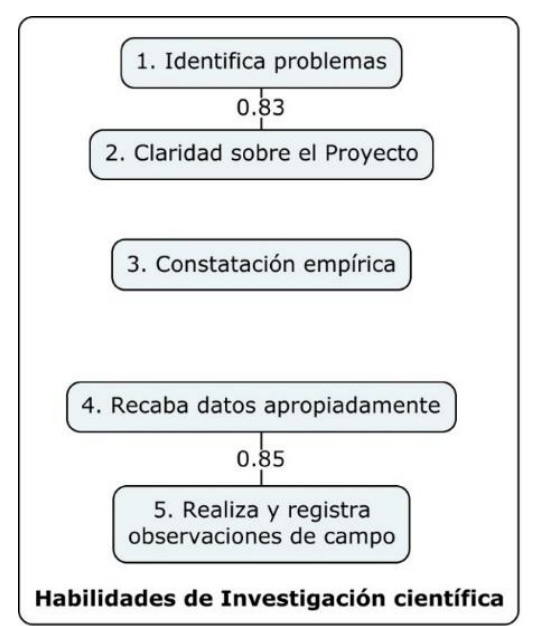

Figura 1. Correlación resultante de analizar las variables de habilidades para la investigación científica donde se muestra desarticulación en algunas de ellas. $r \geq 0.77, \alpha \leq$ 0.01
La comprensión científica de fenómenos y procesos naturales es otra categoría que se relaciona en una etapa posterior con la obtención y sistematización de datos, aquí las 4 variables centrales de la categoría están caracterizadas por: Relacionar sus aprendizajes con la vida cotidiana, el Desarrollar nuevos conocimientos y Utilizar fuentes de información confiable y por último, Planear y llevar a cabo una investigación en el medio local con un propósito definido. Esta última variable se articula con Argumentar utilizando términos científicos de manera adecuada, que a su vez se asocia con Relacionar sus aprendizajes con la vida cotidiana, que es la variable con más relaciones con todas las demás significativas de La comprensión científica como se observa en la Figura 2.

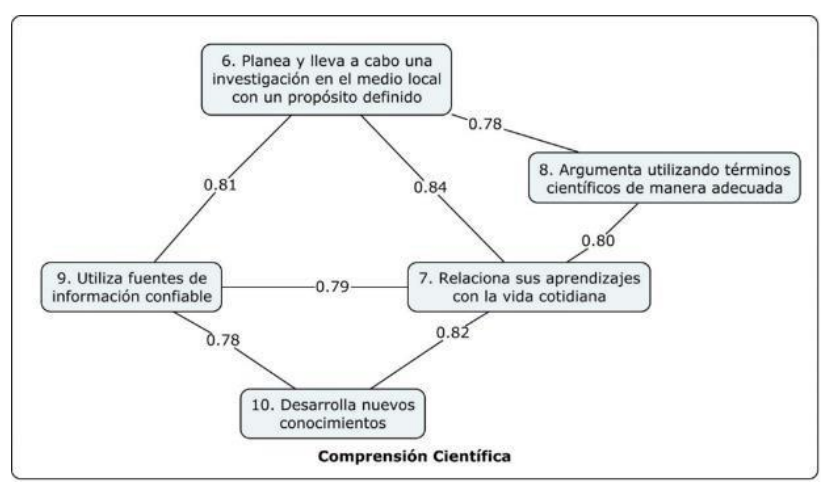

Figura 2. La relación entre todas las variables de la categoría de comunicación de resultados es muy alta en sus valores significativos y superior en todos los casos a una $\mathrm{r} \geq 0.81$

El Manejo de Información técnica y científica resulta fundamental en el proceso de investigación ya que tiene requerimientos de objetividad, racionalidad y verdad, que se manifiestan en las ligas significativas que existen entre las variables de la actividad de investigadores del niño. Éstas variables son: Identificar lo que se necesita saber, Aprender a buscar, Identificar, evaluar, seleccionar, organizar y sistematizar la información recolectada, Apropiarse de la información de manera crítica y Utilizar y compartir información con sentido ético. Obsérvese en la Figura 3 que las variables de esta categoría están integradas o asociadas entre sí. 


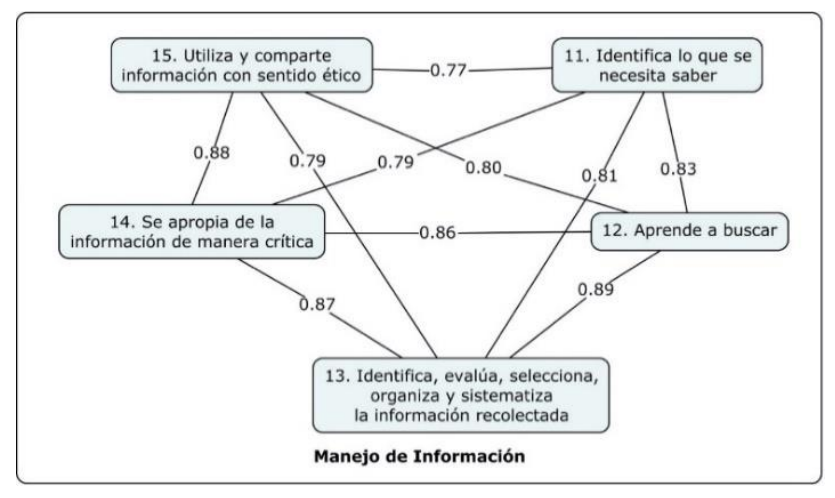

Figura 3. En la categoría del manejo de la información se hace patente la integración de todas las variables que fueron estudiadas en los proyectos de los niños, lo que muestra que este apartado es muy robusto

La Comunicación de resultados del Proyecto es la categoría donde se refleja gran solidez entre los procesos analizados hasta el momento, ya que está compuesta por cinco variables interrelacionadas con valores muy altos de correlación. Aquí se destaca que el niño es capaz de Comunicar sus resultados apropiadamente, Elaborar conclusiones con base en la evidencia disponible, Presentar su cartel o stand con una organización lógica de contenidos, Dar respuestas claras concisas y reflexivas en la presentación oral y Explicar cómo impactará el producto derivado de su proyecto, como se ve en la Figura 4.

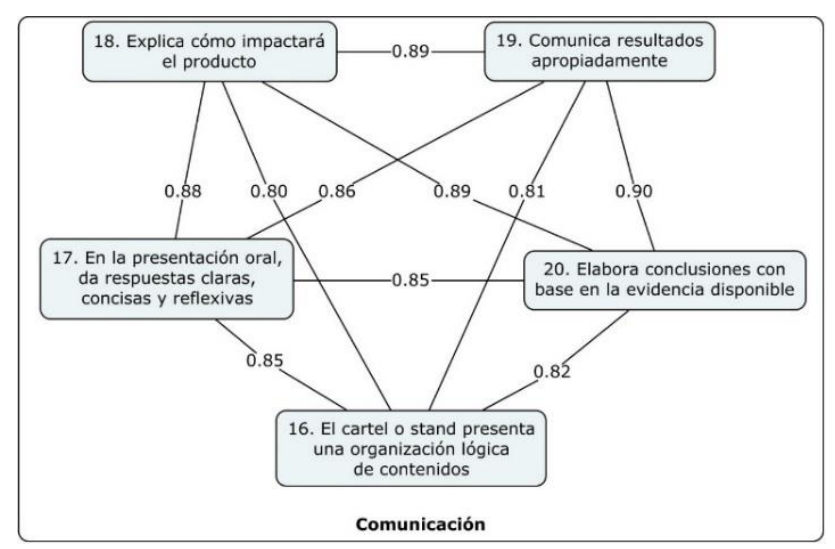

Figura 4. La relación entre todas las variables de la categoría de comunicación de resultados es muy alta en sus valores significativos y superior en todos los casos a una $r \geq 0.81$

\section{Análisis discriminante}

El Análisis discriminante es un tratamiento estadístico que nos permite observar las afinidades de cada uno de las categorías analizadas, con lo que podemos inferir las concordancias, diferencias y analogías de las variables. Hay dos posibles objetivos en un análisis discriminante: encontrar una ecuación predictiva para clasificar nuevos individuos o interpretar la ecuación predictiva para comprender mejor las relaciones que pueden existir entre las variables.

El análisis discriminante encuentra un conjunto de ecuaciones de predicción basadas en variables independientes que se utilizan para clasificar a los individuos en grupos. En este tratamiento se integran todas las variables agrupadas como independientes, a saber: Identifica problemas, Claridad sobre el Proyecto, Constatación empírica, Recaba datos apropiadamente, Realiza y registra observaciones de campo, Planea y lleva a cabo una investigación en el medio local, con un propósito definido, Relaciona sus aprendizajes con la vida cotidiana, Argumenta utilizando términos científicos de manera adecuada, Utiliza fuentes de información confiable, Desarrolla nuevos conocimientos, Identifica lo que se necesita saber, Aprende a buscar, Identifica, evalúa, selecciona, organiza y sistematiza la información recolectada, Se apropia de la información de manera crítica, Utiliza y comparte información con sentido ético, El cartel o stand presenta una organización lógica de contenidos, En la presentación oral, da respuestas claras, concisas y reflexivas, Explica cómo impactará el producto, Comunica resultados apropiadamente, y el Elabora conclusiones con base en la evidencia disponible.

De acuerdo con el análisis discriminante podemos afirmar que por municipio resulta interesante, dado que Monclova, Ramos Arizpe y Saltillo tienen un desarrollo semejante al de Torreon que por sí mismo tiene mayor fortaleza en la semejanza de procedimientos de investigación que hicieron en los proyectos como se observa en la Tabla 3. 
Tabla 3. Tabla de clasificación según Municipio. Podemos observar que los proyectos de la ciudad de Torreón tienen mayor consistencia en su presentación de acuerdo con los valores obtenidos de 89.13 de semejanza. La reducción en error de clasificación debido a las medias fue X's = 53 0\%, con Regresión Múltiple

\begin{tabular}{|l|r|r|r|r|r|}
\hline \multicolumn{7}{|c|}{ Actual } & Monclova & Predicción & & \\
Ramos Arizpe & Saltillo & Torreón & Total \\
\hline Monclova & 15.38 & 15.38 & 15.38 & 2.17 & 2.86 \\
\hline Ramos Arizpe & 0.00 & 0.00 & 0.00 & 0.00 & 2.86 \\
\hline Saltillo & 7.69 & 7.69 & 7.69 & 8.70 & 25.71 \\
\hline Torreón & 76.92 & 76.92 & 76.92 & 89.13 & 68.57 \\
\hline Total & 100 & 100 & 100 & 100 & 100 \\
\hline
\end{tabular}

\section{Análisis factorial}

Las principales aplicaciones de las técnicas analíticas del Análisis factorial son: (1) para reducir el número de variables y (2) para detectar la estructura en las relaciones entre las variables, eso es clasificar variables. Se aplica el análisis factorial como un método (exploratorio) de la reducción de datos o de detección de la estructura. El Análisis de Factores utilizando aquí es el método Centroide que integra los factores con un eigenvalor $>1, y$ separa dos factores bien diferenciados que explican el $76.14 \%$ de la variabilidad total del fenómeno, al seleccionar diferentes variables con una carga factorial $\geq 0.70$ (Tabla 4).

Tabla 4. Dos factores extraídos por el método de Centroide que explican en su conjunto el $76.14 \%$ de la variabilidad total del fenómeno.

\begin{tabular}{|c|c|c|c|c|}
\hline & $\begin{array}{l}\text { Eigen } \\
\text { valor }\end{array}$ & $\begin{array}{c}\% \text { de la } \\
\text { variabilidad }\end{array}$ & $\begin{array}{l}\text { Eigenvalor } \\
\text { Acumulativo }\end{array}$ & $\begin{array}{c}\% \\
\text { Acumula } \\
\text { tivo }\end{array}$ \\
\hline $\begin{array}{l}\text { Factor } 1 \text { Formación } \\
\text { científica básica }\end{array}$ & 13.25 & 66.23 & 13.25 & 66.23 \\
\hline $\begin{array}{l}\text { Factor } 2 \text { Socialización de } \\
\text { la Investigación }\end{array}$ & 1.98 & 9.91 & 15.23 & 76.14 \\
\hline
\end{tabular}

El Factor 1, que denota la Formación científica básica, explica el $66.23 \%$ de la varianza con 8 variables, lo que denota la parte fundamental de su preparación personal para aplicar metodologías científicas en su formación (Tabla 5).

Tabla 5. Factor 1 Formación científica básica

\begin{tabular}{|l|r|}
\hline \multicolumn{1}{|c|}{ Variables } & Factor 1 \\
\hline 1. Identifica problemas & 0.79 \\
\hline 2. Claridad sobre el Proyecto & 0.86 \\
\hline 4. Recaba datos apropiadamente & 0.77 \\
\hline 5. Realiza y registra observaciones de campo & 0.79 \\
\hline $\begin{array}{l}\text { 6. Planea y lleva a cabo una investigación en } \\
\text { el medio local, con un propósito definido }\end{array}$ & 0.74 \\
\hline $\begin{array}{l}\text { 7. Relaciona sus aprendizajes con la vida } \\
\text { cotidiana }\end{array}$ & 0.83 \\
\hline 9. Utiliza fuentes de información confiable & 0.72 \\
\hline 10. Desarrolla nuevos conocimientos & 0.80 \\
\hline
\end{tabular}

El factor dos fue denominado como socialización de la investigación, ya que está integrado por seis variables relacionadas con la proyección y comunicación social de su proyecto. Explica el $9.91 \%$ de la variabilidad total de fenómeno (Tabla 6).

Tabla 6. Factor 2 Socialización de la Investigación

\begin{tabular}{|c|c|}
\hline Variables & $\begin{array}{l}\text { Factor } \\
2\end{array}$ \\
\hline $\begin{array}{l}\text { 14. Se apropia de la información de manera } \\
\text { crítica }\end{array}$ & 0.74 \\
\hline $\begin{array}{l}\text { 16. El cartel o stand presenta una } \\
\text { organización lógica de contenidos }\end{array}$ & 0.80 \\
\hline $\begin{array}{l}\text { 17. En la presentación oral, da respuestas } \\
\text { claras, concisas y reflexivas }\end{array}$ & 0.88 \\
\hline 18. Explica cómo impactará el producto & 0.88 \\
\hline 19. Comunica resultados apropiadamente & 0.90 \\
\hline $\begin{array}{l}\text { 20. Elabora conclusiones con base en la } \\
\text { evidencia disponible }\end{array}$ & 0.89 \\
\hline
\end{tabular}

Es necesario denotar que algunas variables quedan fuera del proceso que utilizan los niños estudiados de Cuarto, Quinto y Sexto año de primaria. Una observación detallada de las variables excluidas seguramente podrá sugerirnos que su comprensión y utilización requieren algo más de maduración cognitiva de los niños, que en ese momento están comenzando a utilizar el pensamiento abstracto rudimentario. Estas variables son: Constatación empírica, Argumenta utilizando términos científicos de manera adecuada, Identifica lo que se necesita saber, Aprende a buscar, Identifica, evalúa, selecciona, organiza y sistematiza la información recolectada y Utiliza y comparte información con sentido ético.

\section{Conclusiones}

La Recodificación de los datos según la metodología de Investigació Mèdica, I. M. (1996), aplicada a las 24 variables (Categorías y variables simples) con 105 casos permitió tratamientos de frecuencias y porcentajes, así como otros análisis multivariados, con lo cual se exploró y explotó más la información. 
Las calificaciones más altas de los niños corresponden a la categoría de Comunicación, específicamente las variables de la Presentación oral, donde dan respuestas claras, concisas y reflexivas, y en la variable que califica que su cartel o stand en la feria presenta una organización lógica de contenidos. Con valores debajo de la normalidad a una desviación estándar encontramos con las más bajas calificaciones las variables que miden que el niño Recaba datos apropiadamente y que Realiza y registra observaciones de campo. Esto resulta interesante ya que ambas son actividades previas a las pruebas experimentales.

La categoría de Habilidades Investigación Científica denota que los elementos de la formación de los niños no están articulados entre sí como fuera deseable para que pudiéramos considerarlos una plataforma de desarrollo posterior del investigador, $L a$ comprensión científica tiene como núcleo el Relacionar sus aprendizajes con la vida cotidiana, que se integra con todas las otras variables de esta categoría con los mayores valores de correlación.

En la categoría del manejo de la información se hace patente la integración de todas las variables que fueron estudiadas en los proyectos de los niños, lo que muestra que este apartado es muy robusto,

La relación entre todas las variables de la categoría de comunicación de resultados es muy alta en sus valores significativos y superior en todos los casos a una $r \geq 0.81$.

El análisis discriminante por municipio resulta interesante, dado que Monclova, Ramos Arizpe y Saltillo tienen un desarrollo semejante al de Torreón que por sí mismo tiene mayor fortaleza en la semejanza de procedimientos de investigación que hicieron en los proyectos.
En el análisis factorial se denota la Formación científica básica de los niños está compuesta por 8 variables del formato de evaluación, Identifica problemas, tiene Claridad sobre el Proyecto, Recaba datos apropiadamente, Realiza y registra observaciones de campo, Planea y lleva a cabo una investigación en el medio local, con un propósito definido, Relaciona sus aprendizajes con la vida cotidiana, Utiliza fuentes de información confiable, y Desarrolla nuevos conocimientos, lo que denota la parte fundamental de su preparación personal para aplicar metodologías científicas en su formación.

Este análisis indica también que la socialización de la investigación en el niño depende de otras variables que son: Se apropia de la información de manera crítica, El cartel o stand presenta una organización lógica de contenidos, En la presentación oral, da respuestas claras, concisas y reflexivas Explica cómo impactará el producto, Comunica resultados apropiadamente y Elabora conclusiones con base en la evidencia disponible.

\section{Referencias}

Batanero, C. (2013). La comprensión de la probabilidad en los niños: ¿qué podemos aprender de la investigación? In J. A. Fernandes, p. F. Correia, m. H. Martinho, \& f. Viseu, (eds..) (2013). Atas do III Encontro de Probabilidades e Estatística na Escola (Pp. 114). Braga: Centro De Investigação Em Educação Da Universidade Do Minho. https://www.researchgate.net/publication/27345 6653_LA_COMPRENSION_DE_LA_PROBA BILIDAD_EN_LOS_NINOS_QUE_PODEMO S_APRENDER_DE_LA_INVESTIGACION

Charpak, Georges; Léna, Pierre; Quéré, Yves. Los niños y la ciencia, La aventura de La mano en la masa. 2006. Educación pedagogía. EDITORIAL Siglo XXI de España Editores. 240 pp. ISBN: 978-987-1220-47-2, EAN: 9789871220472.

Cuevas, A., Hernández, R., Leal, B. E. y Mendoza, C. P. (2016). Enseñanza aprendizaje de ciencia e investigación en educación básica en México. Revista Electrónica de Investigación Educativa, 18(3), 187-200. Recuperado de http://redie.uabc.mx/redie/article/view/1116. 
De Álvaro Marciel, Patricia. El descubrimiento de los fenómenos meteorológicos en educación infantil. Una propuesta experimental. Tabanque, 30 (2017) p. 75-94 ISSN: 02147742.

DOI:

https://doi.org/10.24197/trp.30.2017.75-94

Frutos Marazuela, M. de. (2013). Aprendizaje por indagación: la luz. http://uvadoc.uva.es/handle/10324/4363

Investigación Médica, I. M. (1996). Manual de puntuación de la versión española del Cuestionario de Salud SF-36. www.rediryss.net

LeaI Pérez, Brenda Elizabeth; Hernández Sampieri, Roberto \& Hernández Bastida, Anilu. Actitudes asociadas a la ciencia en la Educación Básica en México. México. Estudios en Ciencias Sociales y Administrativas de la Universidad de Celaya. Enero-Diciembre 2014. Vol. 4 Num. 1. ISSN: 2007-8242. pp. 41-57.

Massarani, L. (2007). Reflexiones sobre la divulgación científica para niños. Quark, 5. http://quark.prbb.org/17/017040.htm

Postigo Fernández, David \& Greca, Ileana María. Uso de la metodología de la indagación para la enseñanza de nociones sobre fuerzas en primer ciclo de la escuela primaria. Año Revista Enseñanza de la Física. Vol. 26, No. Extra, Dic. 2014, 265-273. https://revistas.unc.edu.ar/index.php/revistaEF/

Sánchez A.M.: La divulgación de la ciencia como literatura, Ciudad de México, Universidad Autónoma de México, 1998.

Shamos, Morris H. The Myth of Scientific Literacy, New Jersey, Rutgers University Press, 1995. ISBN-10: 0813521963, ISBN-13: 9780813521961. 279 pp. London. 